\title{
Multimodal Biometric Recognition Using Iris and Face Features
}

\author{
Sulaiman ALSHEBLI ${ }^{a, 1}$, Fatih KURUGOLLU ${ }^{\mathrm{a}}$ and Mahmoud SHAFIK ${ }^{\mathrm{a}, 2}$ \\ ${ }^{a}$ University of Derby, Derby, United Kingdom
}

\begin{abstract}
Multimodal biometrics has recently gained interest over single biometric modalities. This interest stems from the fact that this technique offers improvements in recognition and more security. In this ongoing research programme, we propose a new feature extraction technique for a biometric system based on face and iris recognition. The extraction of iris and facial features is performed using the Discrete Wavelet Transform combined with the Singular Value Decomposition. Merging the relevant characteristics of the two modalities is used to create a pattern for each individual in the dataset. The evaluation process is performed using two datasets (i.e., Faces94 Faces dataset and IIT Delhi Iris dataset). The experimental results carried out in this programme showed the robustness of the proposed technique.
\end{abstract}

Keywords. Face, Iris, Multimodal Biometrics, recognition, DWT.

\section{Introduction}

Biometric systems are tools used to automatically and accurately recognizing people. This recognition is usually based on unique physiological characteristics such as iris, face, and fingerprints, or behavioural characteristics such as signature and gait [1]. Nowadays, the growing demand for reliable and secure recognition systems, used in many fields, proves that more attention needs to be paid to biometrics. These systems are used in many areas such as military, banking, passport verification, airports, buildings, mobile phones, and identity cards [2]. Many biometric systems are based on a single characteristic of the human body. These systems have many limitations related to this single characteristic such as noise, intra-class variation, and identity fraud [2][3].

To overcome these problems, multimodal biometrics was developed. This technique consists of a combination of information from several biometric sources. Multiple biometric images obtained from different sensors can be combined at a different level: (i) sensor level: a data obtains from different sensors is combined and formed as a new dataset and further we create a feature extraction vector. (ii) feature level: at this level, we first extract features from different obtained biometric images and combine them to create a new feature vector. (iii) score level: at this level, different characteristics are extracted, compared individually and a combined match score is obtained. In this work, we are interested in feature-level fusion. The recognition is based on a comparison between the test sample and the template stored in the database as an indicator of similarity for the modalities. A biometric system has two phases, training, and

\footnotetext{
${ }^{1}$ Sulaiman ALSHEBLI. sulaimanalshebli@gmail.com

${ }^{2}$ Mahmoud Shafik.mshafik@derby.ac.uk
} 
recognition. For the training phase, the biometric modality is captured and processed using specific algorithms, to obtain a reference biometric template for each user which is stored in a database. For the recognition phase, a biometric sample is captured and processed as in the training phase and compared with the biometric templates stored in the database. The result is either a match found (if the user is found in the database) or not recognized [4]. The main contribution of this research programme is to pre-process the images before exposing them to the recognition model. This model exploits the performance of the Discrete Wavelet Transform (DWT) method for feature extraction and then uses the Singular Value Decomposition (SVD) method to reduce the size of the feature space. The Euclidean distance method was used to find the match in the database.

The rest of the paper is organized as follows: section two covers an investigation into the state-of-the-art of multimodal recognition. Section three introduces the new proposed model for pre-processing, feature extraction, and matching. This is followed with results analysis, and discussion section. The paper concluded with some of the initial finding and potential ongoing work about the research framework programme.

\section{State of the Art of Multimodal Recognition}

The recognition rate of multimodal systems depends on multiple factors, such as the fusion technique, the feature extraction technique, and the classification method.

In recent years, several researchers have focused on the proposal of reliable, multimodal biometric systems. The proposed models have been based on the combination of at least two characteristics. Among the works are those based on face and voice [5][6], face and fingerprint [7], face and palm print [8], fingerprint and iris [9], face and iris [10] [11], or fingerprint and hand geometry [12] [13].

In this work, we have chosen to propose a biometric system based on the face and iris. We made this choice because the face is the natural means of identifying people and the iris is currently considered to be one of the most accurate biometric systems [10] [11].

Eskandri and Toygar proposed to use left and right iris patterns with optimized features of local and global based facial feature extraction methods using Particle Swarm Optimization (PSO) and Bird Swarm Algorithm (BSA) to remove redundant data for the fusion of face-iris multimodal system with Tanh Score Normalization and Weighted Sum Rule fusion method where the weights are also optimized using PSO and BSA [14].

In their article [15], the authors have proposed a Face-iris multimodal biometric scheme based on feature level fusion. They used a 2D Gabor filter with different scales and orientations for feature extraction on the face and iris, then transform them by histogram statistics into an energy-orientation. After that, Principal Component Analysis method (PCA) was used for dimensionality reduction. Finally, they used Support Vector Machine (SVM) for the matching.

In the work [16], the authors proposed a multimodal face-iris biometric system that combines the advantages of score level, feature level and decision level fusion by considering the optimized information of face and iris biometrics at each level of fusion. The optimized output of one fusion level provides appropriate input for the next fusion level to construct a new and efficient scheme. The optimized scores are computed based on the extracted and fused optimized features of the face and iris modalities and finally the optimized decisions are made according to the optimized ROC's obtained from score level fusion. The authors have used the Log-Gabor transform to extract the facial and Iris features and the BSA feature selection algorithm to select the relevant features. 
In the study [17], the authors proposed a weighted score level fusion technique to combine face and iris. They have used the Daugman method for iris recognition where an automatic segmentation is performed using circular Hough transform to localize iris and pupil area and 1D Log-Gabor filters were used to encode the unique features of the iris into a binary template. For face recognition, a PCA based method was used. For the matching, they have used the Hamming distance for iris and the Euclidean distance for faces. The min-max normalization technique has been used for normalizing the matching score of the iris and face recognition. The normalized scores are merged as a single score using the weighted sum rule.

In [18], the authors proposed an Iris and face recognition system based on PCA and Discrete Coefficient Transform (DCT) for facial features extraction, while iris features were extracted using 1D Log-Gabor filters and Zernike moments. They also used feature selection with Genetic Algorithms (GA) and scores level fusion with SVM.

In another work [2], the authors proposed a Face-Iris multimodal biometric system based on hybrid level fusion. For the features extraction, they used multi-resolution twodimensional Log-Gabor filter combined with spectral regression kernel discriminant analysis. For the matching, they used the Euclidean distance.

In a recent work [11], the authors presented a Face-Iris multimodal biometric identification system based on multi-resolution 2D Log-Gabor filter for iris features extraction and the Singular Spectrum Analysis (SSA) combined with the Normal Inverse Gaussian (NIG) statistical features derived from wavelet transform for the facial features extraction. The matching was performed using the Fuzzy K-nearest Neighbor (FK-NN). Works in the literature have shown that the combination of biometric templates at different fusion levels and using different feature extraction techniques improves the accuracy of the system. The cited works use different datasets to evaluate their algorithms such as: CASIA iris dataset, IIT Delhi Iris dataset, ORL face dataset, FERET face dataset, and Face94 face dataset. These works generally use two different algorithms for feature extraction of the face and Iris which makes the system more complex. The goal of this work is to propose a single algorithm for the extraction of features on the face and iris.

\section{The New Proposed Model for Multimodal Recognition}

In this paper, we will focus on face-iris multimodal biometric system based on DWT and SVD for feature extraction and a system having a feature level fusion. The proposed system is described and detailed in this section.

Figure 1 illustrates the proposed system. A dataset containing images of people's faces and irises is required to extract features. The dataset must contain a set of images of the faces and irises of the same person. The images first go through a pre-processing step for image normalization, then feature extraction is done. A feature vector of each person's facial and iris features is stored in a database. This vector will be used later in the matching step. Matching is a comparison operation using the Euclidean distance to find the best match in the dataset. 
Figure 1. Proposed model

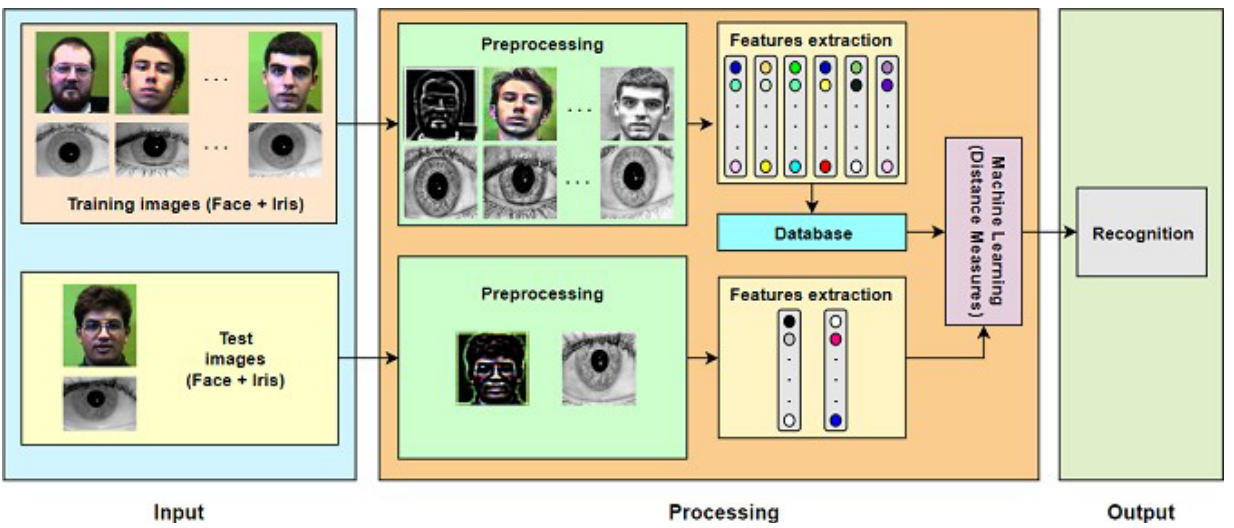

\subsection{Preprocessing}

Before doing image processing, one usually goes through a pre-processing or data cleaning step before building the intelligent model. The purpose of this step is to prepare the images to facilitate their analysis and computer processing. The choice of preprocessing method depends on the quality and nature of the data. In this work, we used two preprocessing methods, namely contrast and Gamma correction.

\subsection{Feature extraction}

Feature extraction prior to matching is an essential task. Feature extraction methods take out the most representative and relevant features of images to achieve a high matching score. In this research we have performed a static face and iris recognition based on the Extracted Feature Vectors obtained from the Discrete Wavelet Transform (DWT). In our proposed experiments, the Singular Value Decomposition (SVD) is applied on each sub image and its dominant features are extracted and stored inside the feature vector. We have compared our approach with three other feature extraction techniques: Discrete Cosine Transform (DCT) [19], Hough Transform (HT) [26], and Malakooti Transform (MT) [27].

\subsubsection{Discrete Wavelet Transform}

Discrete Wavelet Transform (DWT) [20] is an orthogonal transform similar to the Discrete Cosine Transform and an important multiresolution analysis tool. The DWT has been commonly applied to signal processing [21], video compression [22], speech recognition [23], image analysis and feature extraction [24], and various classification systems [25]. The DWT provides sufficient information both for analysis and synthesis of the original image, with a significant reduction in the computation time.

The basic idea of DWT is to provide the time-frequency representation by decomposing the image using DWT into $\mathrm{N}$ levels using filtering and decimation to obtain the approximation and detailed coefficients, then, extracting the features from the DWT coefficients. 
In this study, we used the DWT for feature extraction. The first level DWT divides the input image into four sub-band images. Each sub-band image contains one of high frequency bands and low frequency bands: LL, LH, HL, and HH, where LL denote a low frequency sub-band, LH a horizontal high frequency sub-band, HL vertical high frequency sub-band, and $\mathrm{HH}$ a diagonal high frequency sub-band. Here, the second level DWT decomposition LL2 sub-band image is important for us to reduce the input image size and extract features to be saved in the feature vector. Each time we apply DWT on the input image, the LL sub-band will be one-half of the input image. Thus, when we apply two-level DWT on the input image the size of LL sub-band would be $1 / 4$ of the input image dimension. We have selected the LL2 band because it contains the most useful features of the input image while the other three bands can be ignored.

\subsubsection{Singular Value Decomposition}

Singular Value Decomposition (SVD) is one the strongest mathematical tools that can be used to decompose any square or nonsquare matrix, A, into the multiplication of two unitary matrices $\mathrm{U}$ and $\mathrm{V}$ and one Diagonal matrix $\Sigma$. This technique from the linear algebra can be used to automatically perform dimensionality reduction. SVD is a matrix decomposition method for reducing a matrix to its constituent parts. The SVD is used widely both in the calculation of other matrix operations, such as matrix inverse, but also as a data reduction method in machine learning. SVD can also be used in least squares linear regression, image compression, and denoising data.

Given a nxm matrix A, the SVD of A is:

$$
A=U \cdot \Sigma \cdot \mathrm{V}^{\mathrm{T}}
$$

Where the diagonal values in the $\Sigma$ matrix are known as the singular values of the original matrix A. The columns of the $U$ matrix are called the left-singular vectors of $A$, and the columns of $\mathrm{V}^{\mathrm{T}}$ are called the right-singular vectors of $\mathrm{A}$ where $\mathrm{T}$ is a superscript.

SVD can be thought of as a projection method where data with m-columns (features) is projected into a subspace with $\mathrm{m}$ or fewer columns, whilst retaining the essence of the original data.

\subsection{Matching}

Image matching is an important concept in computer vision and object recognition. In face and/or iris recognition, find a matching means that the system recognize the person, otherwise this person is not registered in the database.

In this work, we carried out a comparative study between three matching methods: the Euclidean distance (ED), the Manhattan distance (MD), and the Cosine similarity (CS).

\subsection{Face and iris recognition process}

In the face and iris recognition process, if the user chooses to do a training on a dataset he must first choose whether or not to do a pre-processing and which method to use 
(Gamma Correction or Contrast Enhancement). After that, he selects the matrix size, this value is used to divide the input image in several blocks $(2,4,8,16,32,64,128$, or 256). Then, experiments using some combinations (DWT+SVD, DCT + SVD, MT+SVD, $\mathrm{HT}+\mathrm{SVD}$ ) are done at the end of the pre-processing and the division steps.

For the DWT+SVD, DWT extraction is carried out by a two-level wavelet decomposition by the Haar wavelet. We have applied the SVD on low-frequency LL sub-bands of the input image and extracted the singular values of Red, Green, and Blue Low-frequency LL sub-bands. Once the singular values of each low-frequency LL subband are calculated using the SVD formula, the result will be appended at the end of the corresponding feature vector and the process of the feature extraction is completed. We applied the same dimensionality reduction process on DCT.

For HT and MT extraction, we need to calculate the HT and MT matrices. For the MT, we have used the author's recommended values of $a$ and $b(a=1, b=2)$. The SVD decomposition of MT/HT is calculated by an element-by-element multiplication of the MT/HT matrix with the image block. This whole process is then applied to the face and iris image.

After this feature extraction step, the feature vectors (of the face and the iris) are fused and stored in a database which will be used later in the matching. Each feature vector (face and iris) is obtained by the concatenation of the S, V, and D component).

In the test phase, the images proposed to the system (facial and iris images) go through the same processing and the resulting feature vector will be compared to the stored features database to find the best match using a distance measure (Euclidean, Manhattan, or Cosine).

\section{Experimental Results Analysis and Discussions}

In this study we used two public datasets widely used in the state of the art, the first is Faces94 which contains facial images [28] and the second is IIT Delhi Iris dataset [29] which contains iris images. We grouped the two datasets so that we had a single dataset containing face and iris images for each person. The irises do not really belong to the people on the faces, but we did this operation to test the different algorithms on multimodal classification (face and iris). The final used dataset contains 150 folders representing individuals. Each folder contains two sub-folders named "Face" and "Iris" which contain the images of faces and irises respectively.

For comparison purposes, we conducted experiments in two stages. The first is a comparison between different feature extraction techniques (DWT+SVD, DCT+SVD, $\mathrm{HT}+\mathrm{SVD}, \mathrm{MT}+\mathrm{SVD}$ ) with different parameters including matrix size, pre-processing technique, and distance measure. Experiments using 1500 images from the Face and Iris dataset were done. First, we have compared the different combinations of our proposed algorithms (DWT, DCT, HT, and MT combined with SVD), the different matrix sizes (32, 64, 128, and 256), the preprocessing methods (contrast and Gamma correction), and the distance measures (Euclidean, Manhattan, and Cosine).

The goal is to visualize and compare the accuracy of each combination to perform a comparison with the state-of-the-art methods in multimodal biometry.

By analyzing the accuracy of different combinations with different distances, it may be observed that our multimodal algorithm (DWT+SVD) gives the best result in the test especially when the matrix size is equal to 128 and a contrast pre-processing is applied with the Euclidean distance as a matching metric (see Table 1). 
Table 1. Best obtained results from different combinations

\begin{tabular}{|c|c|c|c|c|}
\hline Distance & Method & Matrix size & Pre-proc. & Face \& Iris \\
\hline Euclidean & DWT+SVD & 128 & Contrast & 98.90 \\
\hline Manhattan & DWT+SVD & 64 & Contrast & 94.18 \\
\hline Cosine & DWT+SVD & 128 & Contrast & 96.36 \\
\hline
\end{tabular}

The DWT gives the best results, this can be explained by the fact that DWT is better than DCT in terms of time and frequency resolution. Its coefficients are calculated by performing the successive Low pass and High pass filter on the Discrete-Time samples. DWT is also better than HT and MT since it selects only the LL2 band which contains the most useful features of the input image. Euclidean distance gives the best results compared to the Cosine and Manhattan distance. Manhattan distance is usually preferred over the Euclidean distance when there is high dimensionality in the data [30].

In our study, the use of SVD with DWT reduces the size of the features vector, which makes the Euclidean distance more suitable for this case.

Table 2. Comparison results

\begin{tabular}{|c|c|c|c|}
\hline Method & Feature extraction & Matching & Acc. \\
\hline $\begin{array}{l}\text { G. Huo et al. } \\
\text { [15] (2015) }\end{array}$ & $\begin{array}{l}\text { 2D Gabor filter with different scales and orientations, then } \\
\text { transform them by histogram statistics into an energy- } \\
\text { orientation. PCA method is used for dimensionality reduction. }\end{array}$ & $\begin{array}{l}\text { Support Vector } \\
\text { Machine (SVM) }\end{array}$ & 97.81 \\
\hline $\begin{array}{l}\text { Y. Bouzouina et } \\
\text { al. [18] (2017) }\end{array}$ & $\begin{array}{l}\text { PCA and discrete coefficient transform (DCT) for facial features. 1D } \\
\text { Log-Gabor filter method and Zernike moment for iris features. } \\
\text { Genetic algorithm (GA) is used for dimensionality reduction. }\end{array}$ & $\begin{array}{l}\text { Support Vector } \\
\text { Machine (SVM) }\end{array}$ & 96.72 \\
\hline $\begin{array}{l}\text { B. Ammour et } \\
\text { al. [2] (2018) }\end{array}$ & $\begin{array}{l}\text { Two-dimensional Log-Gabor filter combined with spectral } \\
\text { regression kernel discriminant analysis. }\end{array}$ & $\begin{array}{l}\text { Euclidean } \\
\text { Distance }\end{array}$ & 97.45 \\
\hline $\begin{array}{l}\text { B. Ammour et } \\
\text { al. [11] (2020) }\end{array}$ & $\begin{array}{l}\text { The dataset is pre- processed using the histogram equalization } \\
\text { then the features are extracted from face images using singular } \\
\text { spectrum analysis (SSA) and normal inverse Gaussian (NIG) } \\
\text { combined with statistical features of wavelet. Feature } \\
\text { extraction from iris images was performed using multi- } \\
\text { resolution 2D Log-Gabor filter and spectral regression kernel } \\
\text { discriminant analysis (SRKDA) }\end{array}$ & $\begin{array}{c}\text { Fuzzy } \\
\text { K-Nearest } \\
\text { Neighbor } \\
\text { (FK-NN) }\end{array}$ & 98.18 \\
\hline $\begin{array}{l}\text { Our } \\
\text { method }\end{array}$ & $\begin{array}{l}\text { The dataset is pre- processed using the contrast method then } \\
\text { the feature extraction is made by DWT and SVD with the matrix } \\
\text { size equal to } 128 .\end{array}$ & $\begin{array}{l}\text { Euclidean } \\
\text { Distance }\end{array}$ & $\underline{98.90}$ \\
\hline
\end{tabular}

The second stage of the experiments concerns a comparative study with some state- ofthe-art techniques that address the problem of multimodal classification.

Table 2 shows the results of our proposed method compared with four other techniques. We have implemented our proposed methods and the above-described face and iris 
recognition techniques to judge the outcome of this work. The experiment was done in the same environment and on the same training and test samples (same dataset).

From the results, we can see that techniques using Euclidean distance give better results than techniques using Support vector machine (SVM). Fuzzy k-nearest neighbour (FK$\mathrm{NN}$ ) also gives good results, which means that, for the matching, techniques based on distance give better results than techniques based on classical machine learning. For feature extraction, we can notice that DWT combined with SVD ensures a good characterization of the images and thus a good recognition of people. DWT provides simultaneous spatial and frequency domain information of the image and the SVD select the best features obtained from DWT. The results show that our method outperforms the other cited works.

\section{Conclusion}

In this paper, DWT and SVD-based technique for Multimodal Biometry Recognition have been presented. Experimental results show that the proposed DWT+SVD features are effective and efficient as compared to DCT+SVD, MT+SVD, and HT+SVD because it takes the combined advantages of DWT while estimating the features. It has been proved experimentally that the proposed approach provides an effective (better recognition rate) compared to work carried out in the state-of-the-art techniques on Face/Iris recognition. It has also been proved that distance-based matching techniques gives better results than classical machine learning techniques. In the future work, it is planned to use deep machine learning and compare between the various approach investigated in this ongoing programme.

\section{References}

[1] Eskandari M, Toygar O, Demirel H. A NEW APPROACH FOR FACE-IRIS MULTIMODAL BIOMETRIC RECOGNITION USING SCORE FUSION. International Journal of Pattern Recognition and Artificial Intelligence. 2013;27(03):1356004. Available from: https://doi.org/10.1142/ S0218001413560041.

[2] Ammour B, Bouden T, Boubchir L. Face-Iris Multimodal Biometric System Based on Hybrid Level Fusion. In: 2018 41st International Conference on Telecommunications and Signal Processing (TSP); 2018. p. $1-5$.

[3] Kabir W, Ahmad MO, Swamy MNS. Normalization and Weighting Techniques Based on GenuineImpostor Score Fusion in Multi-Biometric Systems. IEEE Transactions on Information Forensics and Security. 2018;13(8):1989-2000.

[4] Jamdar C, Boke A. Review paper on person identification system using multi-model biometric based on face. Int J Sci Eng Technol Res. 2017;6(3):626-629.

[5] Jain A, Nandakumar K, Ross A. Score normalization in multimodal biometric systems. Pattern Recognition. 2005;38(12):2270 - 2285. Available from: http:/www.sciencedirect.com/science/ article/pii/S0031320305000592.

[6] Lin Hong, Anil Jain. Integrating faces and fingerprints for personal identification. IEEE Transactions on Pattern Analysis and Machine Intelligence. 1998;20(12):1295-1307.

[7] Brunelli R, Falavigna D. Person identification using multiple cues. IEEE Transactions on Pattern Analysis and Machine Intelligence. 1995;17(10):955-966.

[8] Ross A, Jain A. Information Fusion in Biometrics. Pattern Recogn Lett. 2003 Sep;24(13):2115-2125. Available from: https://doi.org/10.1016/S0167-8655(03)00079-5.

[9] Elhoseny M, Essa E, Elkhateb A, Hassanien AE, Hamad A. Cascade Multimodal Biometric System Using Fingerprint and Iris Patterns. In: Hassanien AE, Shaalan K, Gaber T, Tolba MF, editors. Proceedings of 
the International Conference on Advanced Intelligent Systems and Informatics 2017. Cham: Springer International Publishing; 2018. p. 590-599.

[10] Morizet N, Gilles J. A New Adaptive Combination Approach to Score Level Fusion for Face and Iris Biometrics Combining Wavelets and Statistical Moments. In: Bebis G, Boyle R, Parvin B, Koracin D, Remagnino P, Porikli F, et al., editors. Advances in Visual Computing. Berlin, Heidelberg: Springer Berlin Heidelberg; 2008. p. 661-671.

[11] Ammour B, Boubchir L, Bouden T, Ramdani M. Face-Iris Multimodal Biometric Identifica- tion System. Electronics. 2020 Jan;9(1):85. Available from: http://dx.doi.org/10.3390/ electronics 9010085.

[12] Kittler J, Messer K. Fusion of multiple experts in multimodal biometric personal identity verification systems. In: Proceedings of the 12th IEEE Workshop on Neural Networks for Signal Processing; 2002. p. $3-12$.

[13] Feng G, Dong K, Hu D, Zhang D. When Faces Are Combined with Palmprints: A Novel Biometric Fusion Strategy. In: Zhang D, Jain AK, editors. Biometric Authentication. Berlin, Heidelberg: Springer Berlin Heidelberg; 2004. p. 701-707.

[14] Eskandari M, O" nsen Toygar. Selection of optimized features and weights on face-iris fusion using distance images. Computer Vision and Image Understanding. 2015;137:63 - 75. Available from: http://www.sciencedirect.com/science/article/pii/S1077314215000454.

[15] Huo G, Liu Y, Zhu X, Dong H, He F. Face-iris multimodal biometric scheme based on feature level fusion. Journal of Electronic Imaging. 2015;24(6):1 - 10. Available from: https://doi.org/10. 1117/1.JEI.24.6.063020.

[16] Eskandari M. Optimum scheme selection for face-iris biometric. IET Biometrics. 2017 September;6:334-341(7). Available from: https://digital-library.theiet.org/content/ journals/10.1049/ietbmt.2016.0060.

[17] Matin A, Mahmud F, Ahmed T, Ejaz MS. Weighted score level fusion of iris and face to identify an individual. In: IEEE proceeding of the International Conference on Electrical, Computer and Communication Engineering (ECCE); 2017. p. 1-4.

[18] Bouzouina Y, Hamami L. Multimodal biometric: Iris and face recognition based on feature selection of iris with GA and scores level fusion with SVM. In: 2017 2nd International Conference on Bioengineering for Smart Technologies (BioSMART); 2017. p. 1-7.

[19] Ahmed N, Natarajan T, Rao KR. Discrete Cosine Transform. IEEE Transactions on Computers. 1974;C23(1):90-93. In: Furht B, editor. Discrete Wavelet Transform (DWT). Boston, MA: Springer US; 2008. p. $188-188$.

[20] Available from: https://doi.org/10.1007/978-0-387-78414-4_305.

[21] Madhusmita M, Mousumi B, Narayan PD, Kumar MS. A Novel Method for Epileptic EEG Classification Using DWT, MGA, and ANFIS: A Real Time Application to Cardiac Patients with Epilepsy. In: Mallick PK, Balas VE, Bhoi AK, Zobaa AF, editors. Cognitive Informatics and Soft Computing. Singapore: Springer Singapore; 2019. p. 525-534.

[22] Bhardwaj A, Khunteta A. Video watermarking equations using DWT and DCT sub-sub bands for secure transmission over communication channels: A research paper. In: 2017 IEEE International Conference on Power, Control, Signals and Instrumentation Engineering (ICPCSI); 2017. p. 436-440.

[23] Tufekci Z, Gowdy JN. Feature extraction using discrete wavelet transform for speech recogni- tion. In: Proceedings of the IEEE SoutheastCon 2000. 'Preparing for The New Millennium' (Cat. No.00CH37105); 2000. p. 116-123.

[24] Thakral S, Manhas P. Image Processing by Using Different Types of Discrete Wavelet Transform. In: Luhach AK, Singh D, Hsiung PA, Hawari KBG, Lingras P, Singh PK, editors. Advanced Informatics for Computing Research. Singapore: Springer Singapore; 2019. p. 499-507.

[25] Li M, Chen W, Zhang T. Classification of epilepsy EEG signals using DWT-based envelope analysis and neural network ensemble. Biomedical Signal Processing and Control. 2017;31:357 - 365. Available from: http://www.sciencedirect.com/science/article/pii/S1746809416301343.

[26] Hough PVC. METHOD AND MEANS FOR RECOGNIZING COMPLEX PATTERNS.

[27] Malakooti MV, Dobuneh MRN. A lossless digital encryption system for multimedia using orthogonal transforms. In: 2012 Second International Conference on Digital Information and Communication Technology and it's Applications (DICTAP); 2012. p. 240-244.

[28] Spacek L. Collection of Facial Images: Face94. Available from: https://cswww.essex.ac.uk/mv/ allfaces/faces $94 . h$ tml.

[29] Kumar A, Passi A. Comparison and combination of iris matchers for reliable personal authentication. Pattern Recognition. 2010;43(3):1016-1026.

[30] Aggarwal CC, Hinneburg A, Keim DA. On the Surprising Behavior of Distance Metrics in High Di mensional Space. In: Van den Bussche J, Vianu V, editors. Database Theory — ICDT 2001. Berlin, Heidelberg: Springer 